\title{
Opportunities for Practices in Precision Population Health and Personalized Medicine
}

\author{
William W. Au1* \\ 1 University of Medicine, Pharmacy, Science and Technology of Târgu Mureș, Romania; Shantou University Medical \\ College, Shantou, China; and University of Texas Medical Branch, Galveston, Texas, USA
}

During the previous decades, the practice of cancer prevention and intervention has achieved much success at the population level. However, it has become obvious that such achievements at the individual levels have been highly variable and, perhaps, disappointing. Therefore, to significantly improve efficacy, the United States and the European Union have set up priority programs on the development of precision population health and personalized medicine. The emphasis will require a paradigm shift in focusing these activities onto individuals instead of populations. In addition, it changes our traditional approach in conducting basic research and clinical medicine, e.g. by incorporating more genomic information and more personal data into research and practice activities.

Genomic analyses have clearly shown that inherent variations in genes are key factors which determine how individuals respond to toxic substances and therapeutic drugs, therefore their risk for development of cancer and for poor response to therapy. However, mechanisms for these outcomes and their interactions with environmental factors have not been well-characterized. Therefore, cancer risk assessment, and prevention, intervention and prognosis determinations have still mainly been based on population rather than on individual evaluations. For example, lung cancer mortality risk from smoking has been shown to be about 150 per 100,000 individuals who are at 50 years of age (much higher at more advanced ages). The risk determination is well-recognized and have been used to set up smoke-ban policies around the world. However, the risk level has not been effective in convincing smokers to quit the smoking habit because they would probably feel that the risk is small and because they are unlikely to be within the 150 lung cancer mortality cases! Consequently, smoking control has basically relied on the development of smoke-ban policies.
For implementation of precision population health, dynamic changes in our basic research have been initiated. For example, many studies are being conducted by using genomic analysis to more precisely understand mechanisms for development of cancers. These studies have also paved the way for the development of novel biomarkers. Such biomarkers must fulfill requirements to provide functional, quantitative and specific determination of personal risk for development of diseases. Some of them have been focused onto how individual's cell recognizes DNA damage and mounts appropriate responses, a process known as DNA damage and repair response (DDRR). An example for such an assay is the Challenge-comet assay which is developed by Professor Au (Kaina, et al., 2018). In this assay, an individual's DDRR capacity can be quantitatively determined. Investigations have shown that the DDRR capacity is genetically determined, compromised by exposure to toxic substances, associated with development of cancer and associated with poor cancer prognosis (Kaina, et al., 2018). Therefore, individual's DDRR capacity can be used to develop personalized health and disease prevention protocols, e.g. avoidance of exposure to certain toxic substances from which repair of the induced damage is deficient for the specific individuals.

The DDRR capacity data can also be used for precision medicine. For example, cancer patients with low DDRR capacity, e.g. poor repair for radiation-induced DNA damage, may be considered for using alternative (non-radiation dependent) therapeutic protocols. These patients should also be monitored using more specific, i.e. non-traditional, protocols for their response. Furthermore, these patients can also be treated with other DNA damaging drugs which differentiate the DDRR capacity between the normal and the cancer cells to specifically eliminate the cancer cells, therefore achieving better efficacy. 
Novel biomarkers, such as the Challenge-comet assay, should be used in combination with other assays, e.g. DNA methylation and miRNA expression, as well as with bioinformatics information to more precisely predict cancer development and response to therapy. Consequently, these initiatives can dramatically change the evaluation and management of health problems from a population to a personalized basis. Indeed, innovative genomic- and mechanism-based evidence are increasingly used to develop more precise cancer risk assessment, and target-specific and personalized medicine. Successful implementation of personalized medicine is expected to significantly improve health and quality of life, reduce suffering, and improve economy as well as prosperity.

\section{口 CONFLICT OF INTEREST}

None to declare.

\section{DEFERENCE}

Kaina B, Izzotti B, Xu J, Christmann M, Pulliero A, Dobreanu $\mathrm{M}, \mathrm{Au} \mathrm{W}$. Inherent and toxicant-provoked reduction in DNA repair capacity as a mechanism for carcinogenesis and for poor response to therapy. Int J Hygiene Env Health 221:993-1006, 2018. 